\title{
Simplified Multimodal Biometric Identification
}

\author{
Abhijit Shete*, Kavita Tewari* \\ * Department of Electronics \& Telecommunication Engineering, VES Institute of Technology \\ VES Institute of Technology, \\ Hashu Adwani Memorial Complex, Sindhi Society, Chembur, Mumbai-400074, India. \\ Email: abhijit0412@gmail.com
}

\begin{abstract}
Multibiometric systems are expected to be more reliable than unimodal biometric systems for personal identification due to the presence of multiple, fairly independent pieces of evidence e.g. Unique Identification Project "Aadhaar" of Government of India. In this paper, we present a novel wavelet based technique to perform fusion at the feature level and score level by considering two biometric modalities, face and fingerprint. The results indicate that the proposed technique can lead to substantial improvement in multimodal matching performance. The proposed technique is simple because of no preprocessing of raw biometric traits as well as no feature and score normalization.
\end{abstract}

Keywords: Wavelet, Multimodal Fusion, Decidibility Index, EER, ROC, GAR

\section{Introduction}

A number of biometric characteristics are being used in various applications. Each biometric has its pros and cons and, therefore, the choice of a biometric trait for a particular application depends on a variety of issues besides its matching performance. No single biometric is expected to effectively meet all the requirements (e.g. accuracy, practicality, cost) imposed by all applications. In other words, no biometric is ideal but a number of them are admissible. The relevance of a specific biometric to an application is established depending upon the nature and requirements of the application, and the properties of the biometric characteristic.

Several approaches of automatic fingerprint matching have been proposed in the literature. The most popular ones are based on the minutiae pattern of the fingerprint and are collectively called minutiae-based approaches. The most popular approaches to face recognition are based on either (i) the location and shape of facial attributes, such as the eyes, eyebrows, nose, lips, and chin and their spatial relationships, or (ii) the overall (global) analysis of the face image that represents a face as a weighted combination of a number of canonical faces [1].

In this paper performance of the biometric system is improved by fusion techniques [2] [3] and processing steps are reduced by using wavelet based feature extraction method [4] for fingerprint and face biometric. Also use of common similarity mesure [5] eliminates feature level and score level normalization in multimodal biometric fusion. Feature Level Fusion is made easy by using similar techniques for both the biometrics.

\section{Biometric Traits Used and Feature Extraction}

Fingerprint reorganization using minutiae-based approaches are different from one other, most of these methods require extensive preprocessing operations (e.g. orientation flow estimation, ridge segmentation, ridge thinning, minutiae detection) in order to reliably extract the minutia features [6]. They either match directly the fingerprint images [7], or match features extracted from the image by means of certain filtering or transform operations [8], hence their name image-based methods. These approaches require less preprocessing effort than minutiae-based methods but, on the other hand, they have a limited ability to track variations in position, scale, and rotation angle.

Face recognition is a non-intrusive method, and facial attributes are probably the most common biometric features used by humans to recognize one another. 


\subsection{Wavelet Domain Features}

It is well known that fingerprints are quasi-periodic patterns whose dominant frequencies are located in the middle frequency channels. The ridge orientation as well as the ridge spatial frequency in different image regions represent the intrinsic nature of the fingerprint image.

Wavelet transform is a powerful tool for signal analysis and it is widely used in the field of image processing. Let $\Psi(x)$ be mother wavelet, the basis function $\Psi_{a, b}(x)$ can be obtained according to dilation parameter $a$ and translation parameter $b \Psi_{a, b}(x)=2^{-a / 2} \Psi\left(2^{-a}-b\right)$. The one dimensional wavelet transform of signal $f(x) \in L^{2}(R)$ is defined as

$$
W f(x)(a, b)=|a|^{-1 / 2} \int_{R} f(x) \Psi\left(\frac{x-b}{a}\right) d x
$$

The 2-D wavelet transformation is performed by using 1-D wavelet transformation in terms of filtering by rows and columns respectively.

Wavelet filters have been implemented due to their simplicity, suitability and regularity for face recognition using multiresolution approaches. 2-D wavelet is very useful for image processing because the image data is discrete and the spatial-spectral resolution is dependent on the frequency. The wavelet has the property that the spatial resolution is small in lowfrequency bands but large in high-frequency bands. The main reasons for its popularity lie in its complete theoretical framework, the great flexibility for choosing bases and the low computational complexity.

The 2-D wavelet decomposition on $\mathrm{J}$ octaves of a discrete image $A_{0}[n, m]$ represents the image in terms of $3 J+1$ subimages

$$
\left[A_{J},\left\{D_{j}^{1}, D_{j}^{2}, D_{j}^{3}\right\}_{j=1, \ldots ., J}\right]
$$

Where $A_{J}$ is a lowpass approximation of the original image, and $D_{j}^{r}$ are the highpass image details at different scales $2^{j}$ and orientations $r$. Wavelet coefficients of large amplitude in $D_{j}^{1}, D_{j}^{2}$ and $D_{j}^{3}$ correspond, respectively, to vertical high frequencies (horizontal edges), horizontal high frequencies (vertical edges), and high frequencies in both directions.

The normalised $l_{2}$-norm of each wavelet sub-band is computed in order to create a feature vector of length $3 \mathrm{~J}$, as given by Eq.(3)

$$
\left[\left\{e_{j}^{1}, e_{j}^{2}, e_{j}^{3}\right\}_{j=1, \ldots ., J}\right]
$$

Where

$$
e_{j}^{r}=\left\|D_{j}^{r}\right\|_{2} / \sum_{i=1}^{J} \sum_{l=1}^{3}\left\|D_{i}^{l}\right\|_{2}
$$

for all $j=1 \ldots \ldots \ldots$ and $r=1,2,3$

The feature vector represents an approximation of the image energy distribution over different scales $2^{j}$ and orientation $r$. The length of feature vector used in this paper is $l=J * r=5 * 3=$ 15 for both biometric traits. For face images sym 9 wavelet and for finger images $d b 9$ wavelet is used. The scaling and wavelet functions of sym 9 and $d b 9$ is as shown in Figure 1 and Figure 2 respectively. 


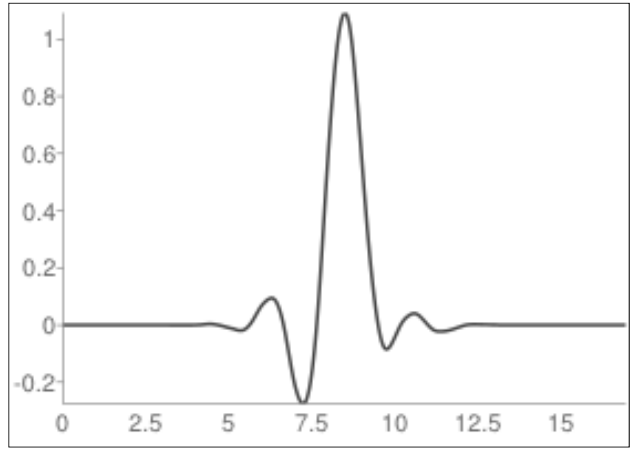

Figure 1(a). Sym9 Scaling Function

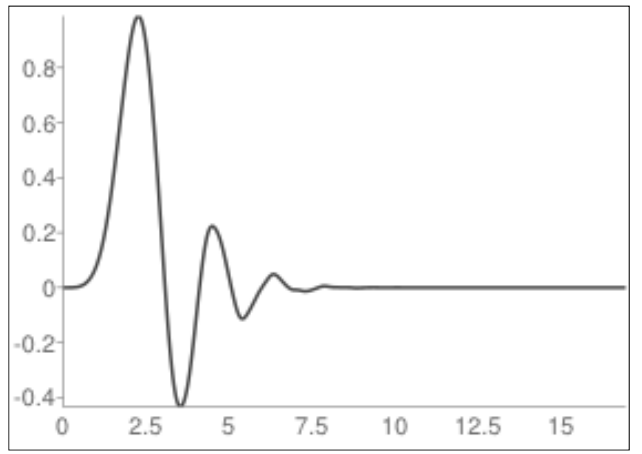

Figure 2(a). db9 Scaling Function

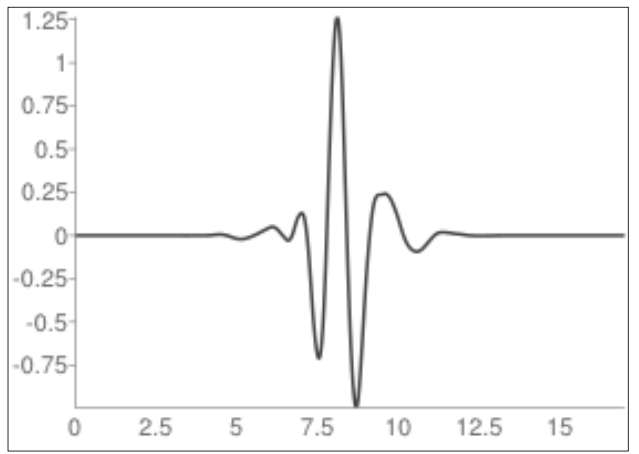

Figure 1(b). Sym9 Wavelet Function

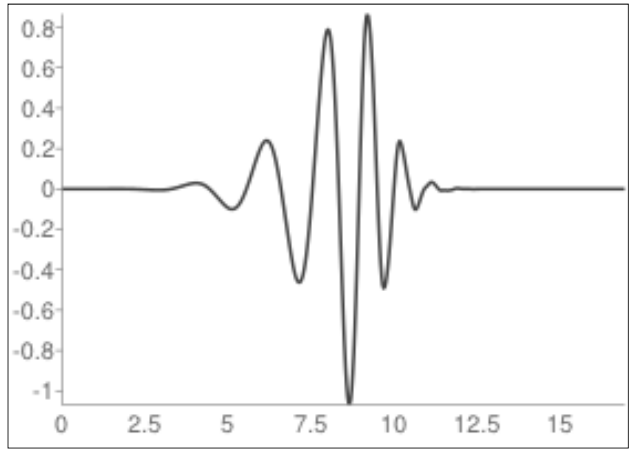

Figure 2(b). db9 Wavelet Function

The intersection operator introduced by Swain and Ballard in [5] is used as a measure of similarity between two feature vectors. If $Q$ and T are the two feature vectors, then measure of similarity between them can be given as

$$
H(Q, T)=\frac{\sum_{i=1}^{3 J} \min \left(Q_{i}, T_{i}\right)}{\min \left(\sum_{i=1}^{3 J} Q_{i}, \sum_{i=1}^{3 J} T_{i}\right)}
$$

$H=1$ Indicates match and $H=0$ indicates non-match condition as shown in Figure 3(a) In this paper, we have examined performance of two fusion techniques, Feature Level Fusion and Match Score Level fusion with three different fusion strategies.

\subsection{Fusion Strategy A - Feature Level Fusion:}

Fusion at the match score, rank and decision levels have been extensively studied in the literature. Fusion at the feature level, however, is a relatively understudied problem [2]. Since the feature set contains richer information about the raw biometric data than the match score or the final decision, integration at this level is expected to provide better authentication results. However, fusion at this level is difficult to achieve in practice because of the following reasons: (i) the feature sets of multiple modalities may be incompatible (e.g., minutiae set of fingerprints and Eigen coefficients of face); (ii) the relationship between the feature spaces of different biometric systems may not be known; (iii) concatenating two feature vectors may result in a feature vector with very large dimensionality leading to the 'curse of dimensionality' wellknown pattern recognition problem; and (iv) a significantly more complex matcher might be required in order to operate on the concatenated feature set.

In the proposed work, wavelet based feature vector and similarity mesure used resolves the problems mentioned above. 
Let $X_{q}=\left\{x_{1}, x_{2}, x_{3} \ldots . x_{l}\right\} \quad$ and $Y_{q}=\left\{y_{1}, y_{2}, y_{3} \ldots . y_{l}\right\}$ where $q=1,2,3 \ldots \ldots . l$ represent the feature vector of the finger and face modalities of a user, respectively. The fused vector $F_{m}$ can be obtained by concatenating individual vector as $F_{m}=\left\{X_{q}, Y_{q}\right\}_{m}$ where $m=2 l$.

\subsection{Fusion Strategy B - Score Level Fusion: (Assignment of Weights based on EER)}

This fusion strategy assigns the weight to each characteristic e.g face and finger, based on their equal error rate (EER). Weights for more accurate characteristic are higher than those of less accurate characteristic. Thus the weights are inversely proportional to the corresponding errors. Let $e_{k}$ be the EER to characteristic $k$, then weight $w_{k}$ associated to characteristic $k$ can be computed by,

$$
w_{k}=\left(\sum_{k=1}^{t} \frac{1}{e_{k}}\right)^{-1} * \frac{1}{e_{k}}
$$

2.4. Fusion Strategy C - Score Level Fusion: (Assignment of Weights based on Decidability Index)

In strategy $\mathrm{C}$, weights are assigned to individual characteristic based on their imposter and genuine scores distributions. The means of these distribution are defined by $\mu_{k}^{I}$ and $\mu_{k}^{G}$ respectively, and standard deviations by $\sigma_{k}^{I}$ and $\sigma_{k}^{G}$ respectively. A parameter Decidability Index $d_{k}$ is used as measure of separation of these two distributions for characteristic $k$ as

$$
d_{k}=\frac{\sqrt{2}\left|\mu_{k}^{G}-\mu_{k}^{I}\right|}{\sqrt{\left(\sigma_{k}^{G}\right)^{2}+\left(\sigma_{k}^{I}\right)^{2}}}
$$

If $d_{k}$ is small, overlap region of two distributions is less. Therefore, weights are assigned to each characteristic proportional to this parameter as,

$$
w_{k}=\left(\sum_{k=1}^{t} d_{k}\right)^{-1} * d_{k}
$$

For both fusion strategies B and C $0 \leq w_{k} \leq 1,(\forall k) ; \sum_{k=1}^{t} w_{k}=1$ and the fused score for user $i$ is computed as,

$$
F_{m}=\sum_{k=1}^{t} w_{k} * S_{k p} ;(\forall p)
$$

In our case $t=2, k=1$ indicates finger and $k=2$ indicates face. $S_{k p}$ indicates match score of $p^{\text {th }}$ pair of $k^{\text {th }}$ characteristic.

\section{Performance Evaluation and Experimental Results}

\subsection{Performance Evaluation}

We performed the experiments on Intel Core2 Duo machine using Matlab (R2010b). The performance of proposed approach is measured in terms of Receiver Operating Characteristic $(R O C)$ curve, which plots Genuine Accept Rate $(G A R)$ against the False Match Rate $(F M R)$ at different thresholds. The FMR, False Non-Match Rate $(F N M R)$ and GAR are given by Eqs. (10)-(12), respectively.

$$
\begin{aligned}
& F M R=\frac{\text { Imposter claims accepted }}{\text { Total } \text { imposter claims }} \times 100 \\
& F N M R=\frac{\text { True claims rejected }}{\text { Total true claims }} \times 100
\end{aligned}
$$


Let $n=$ number of individuals and $m=$ number of images per individual, then number of genuine scores can be obtained as $n m(m-1) / 2$ and imposter scores can be obtained as $n(n-1) m^{2}$ using the same database. For the database used $n=40$ and $m=8$, therefore we get 1120 genuine matching's and 99840 imposter matching's. In this paper we have used 1120 genuine and 6240 imposter pairs for each database. FMR and FNMR are obtained for all thresholds $(t)$ as,

$$
\begin{aligned}
& F M R_{k}(t)=\frac{1}{T_{k}^{l}} \sum_{s=t}^{1} \operatorname{Imposter}(s) \\
& F_{N M R_{k}}(t)=\frac{1}{T_{k}^{G}} \sum_{s=0}^{t} \operatorname{Genuine}(s)
\end{aligned}
$$

Where $T_{k}^{I}$ and $T_{k}^{G}$ are the total number of imposter and genuine matches respectively. Equal Error Rate $\left(E E R_{k}\right)$ is define as the rate at which $F M R_{k}(t)=F N M R_{k}(t)$. In practice the score distributions are not continuous and a crossover point might not exist. In this case, we report the interval as per FVC2000: Fingerprint Verification Competition.

\subsection{Database Used}

The FVC2000-Db1_a fingerprint database [9] contains a total 800 fingerprint images of size 300x300 and 500 dpi resolution from 100 individuals with 8 images per individual, which were captured with low-cost optical sensor "Secure Desktop Scanner" by KeyTronic.

The ORL standard face database [10] consists of 400 face images attained from 40 individuals. Each individual have 10 images of different expression or gesture. The resolution of the image is $112 \times 92$ and the gray scale is 256 . In this work, we have selected 40 individuals and 8 images per individual from each database resulting 320 images per trait.

\subsection{Experimental Results}

The experimental results obtained are shown in Table 1 . The values of EER, $d_{k}$ and GAR are significantly improved in all fusion strategies $A, B$ and $C$ than individual biometric Face and Finger. Among the fusion strategies the EER and $d_{k}$ of strategy A are lower than other strategies, where as GAR of strategy $B$ is higher than strategies A and C.

Table 1. Comparison of EER, $\mathrm{d}_{\mathrm{k}}$ and GAR for different fusion strategies

\begin{tabular}{cccccc}
\hline $\begin{array}{c}\text { Performance } \\
\text { Parameter }\end{array}$ & $\begin{array}{c}\text { Face } \\
\text { alone }\end{array}$ & $\begin{array}{c}\text { Finger } \\
\text { alone }\end{array}$ & $\begin{array}{c}\text { Fusion } \\
\text { Strategy A }\end{array}$ & $\begin{array}{c}\text { Fusion Strategy B } \\
\boldsymbol{W}_{\mathbf{1}}=\mathbf{0 . 4 4 6 2} \\
\boldsymbol{W}_{\mathbf{2}}=\mathbf{0 . 5 5 3 8}\end{array}$ & $\begin{array}{c}\text { Fusion Strategy C } \\
\boldsymbol{W}_{\mathbf{1}}=\mathbf{0 . 4 9 0 3} \\
\boldsymbol{W}_{\mathbf{2}}=\mathbf{0 . 5 0 9 7}\end{array}$ \\
\hline EER & 0.1410 & 0.1750 & 0.0875 & 0.0908 & 0.0899 \\
$\boldsymbol{d}_{\boldsymbol{k}}$ & 61.63 & 59.28 & 119.38 & 117.75 & 119.28 \\
GAR & 37.95 & 29.64 & 65.89 & 66.96 & 66.34 \\
\hline $0.01 \%$ FMR & 37.96 & & \\
\hline
\end{tabular}

The example of performance graphs of fusion strategy $\mathbf{A}$ are shown in Figure 3. Similar graphs can also be obtained for other strategies. 


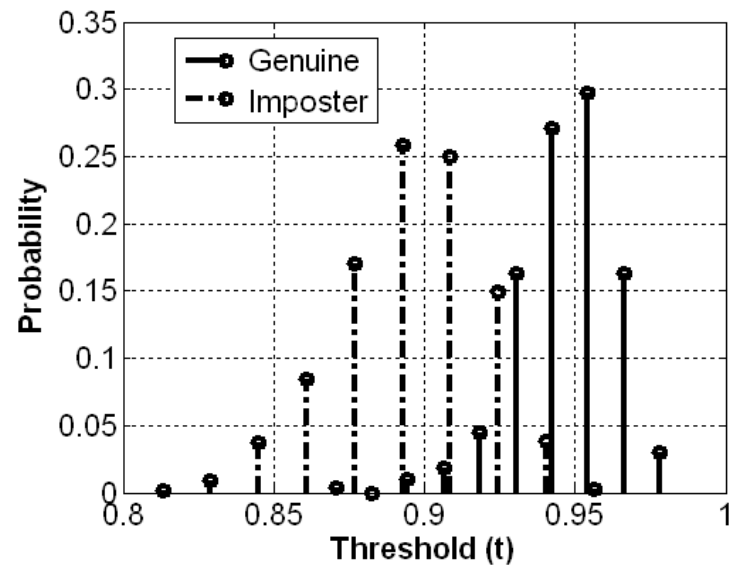

Figure 3(a). Genuine and Imposter Distribution

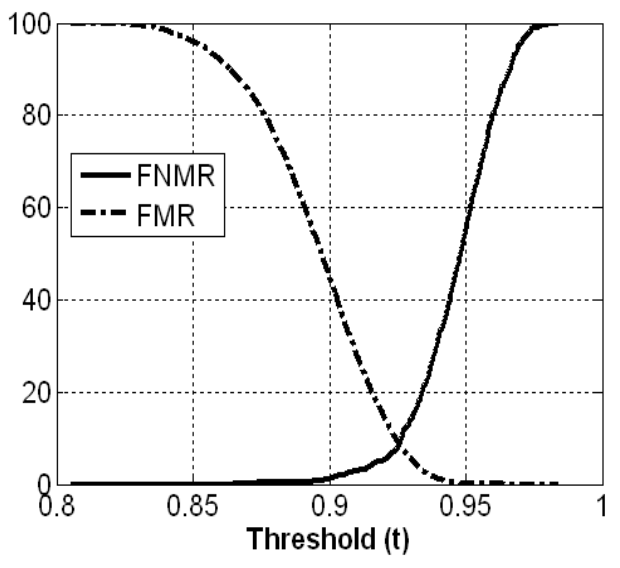

Figure 3(b). FNMR and FMR curves

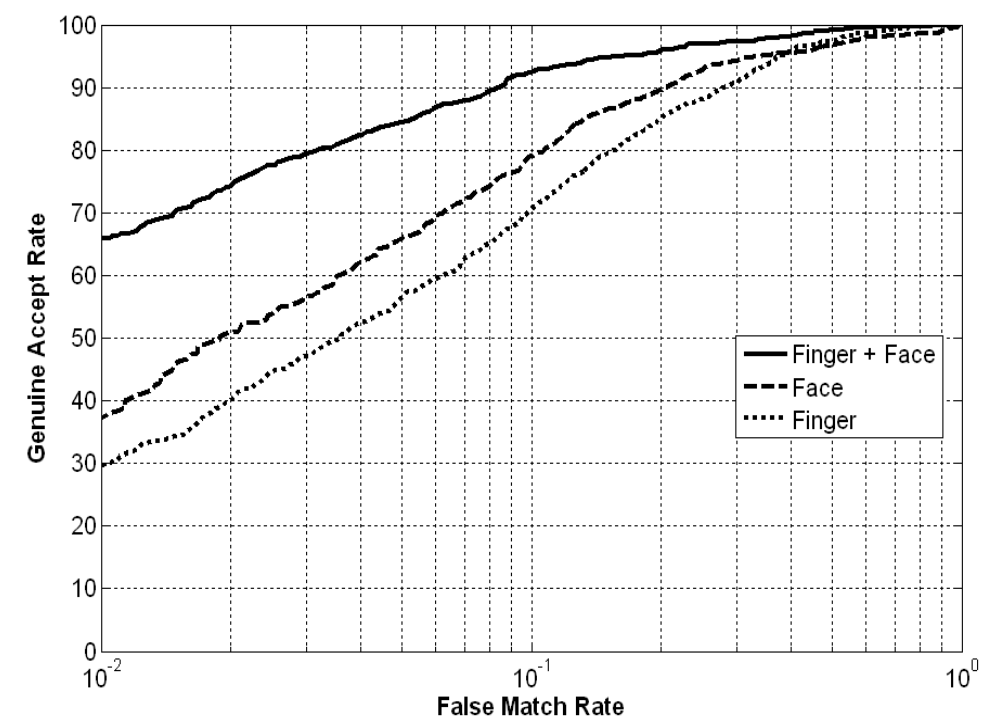

Figure 3(c). ROC curves.

\section{Conclusion}

This paper deals with feature level and score level biometric fusion techniques. The experimental results obtained show that the wavelet features extracted for both face and finger, resolves the problem of compatibility and curse of dimensionality of feature vector. Since in this work, preprocessing of raw biometric traits as well as feature and score normalization is not used, multibiometric identification is simplified. Results show that as per as EER and $d_{k}$ is concerned, feature level fusion, the simplest technique out performs over score level fusion techniques mentioned. GAR of fusion strategy $B$, in which weights are decided by EER is better than other fusion techniques. Since difference between weights $W_{1}$ and $W_{2}$ of fusion strategy $C$ is less than fusion strategy $B, E E R$ and $d_{k}$ value shows improvement for fusion strategy $C$.

\section{References}

[1] Lin Hong and Anil Jain, Fellow. IEEE. "Integrating Faces and Fingerprints for Personal Identification". IEEE Transactions on Pattern Analysis and Machine Intelligence. 1998; 20(12). 
[2] Arun Ross and Rohin Govindarajan, "Feature Level Fusion Using Hand and Face Biometrics", Appeared in Proc. Of SPIE Conference on Biometric Technology for Human Identification II, Orlando, USA. 2005; 5779: 196-204

[3] YN Singh, P Gupta. "Quantitative Evaluation of Normalization Techniques of Matching Scores in Multimodal Biometric Systems". SW Lee and SZ Li (Eds.): ICB2007, LNCS 4642, PP. 574-583, 2007 (c) Springer-Verlag Berlin Heidelberg 2007.

[4] M Tico, P Kuosmanen and J Saarinen. "Wavelet domain features for fingerprint recognition". Electronics Letters. 2001; 37(1).

[5] Swain ML and Ballard DH. "Color indexing". Int, J. Comput.Vis., 1991; 7(I): 11-32

[6] Jain AK, Hong L, pankanti S, and Bolle R. "An identity authentication system using fingerprints", Proc. IEEE. 1997; 85(9): 1364-1388

[7] Willson CL, Watson CT, and Paek EG. "Effect of resolution and image quality on combined optical and neural network fingerprint matching". Patt. Recognit. 2000; 33(Z): 317-331

[8] Lie CJ and Wang SD. "Fingerprint feature extraction using Gabor filters". Eleclron. Lett. 1999; 35(4): 288-290

[9] Finger print database - FVC2000 - Db1_a http://bias.csr.unibo.it/fvc2000

[10] Face Database - ORL (AT\&T) http://www.cl.cam.ac.uk/research/dtg/attarchive/facedatabase.html

[11] Handbook of Fingerprint Recognition. Maltoni D, Maio D, Jain AK and Prabhakar S. Springer, 2009.

[12] Multirate systems and Filter banks, PP Vaidyanathan, Englewood Cliffs; Prentice Hall. 1993.

[13] Handbook of Multi Biometrics, Arun A. Ross ,Karthik Nandakumar .Anil K. Jain. Springer 